\title{
Escuela de Comercio del Consulado Gaditano
}

\author{
M. ㅁ DEL MAR BARRIENTOS MARQUEZ
}

\section{LA FUNDACION}

La implantación de las "enseñanzas de comercio" en el Consulado de Cádiz y demás consulados españoles surge tras la Real Orden de 1799.

El Plan de Estudios para aprender comercio que propone el Consulado gaditano es aprobado por la Real Orden del 11 de mayo de 1803, tras la demanda del Consulado requiriendo dicha aprobación. Se solicitaba que en la nueva Escuela Mercantil se formasen comerciantes instruidos, capaces de ejercer el "noble oficio" del comercio. Su consecución se consideraría un gran éxito para la ciudad, teniendo en cuenta el lugar primordial que seguía ocupando Cádiz en el tránsito comercial, incluso tras la supresión de la Casa de Contratación y la creación del Juzgado de Arribadas.

Se pensaba que esta Escuela produciría enormes ventajas al comercio, por lo que su funcionamiento interesaría al Estado (1). Los solicitantes exponían:

"El comercio era considerado como la verdadera esencia del modo de subsistir, el ignorarlo sería el peligro mayor en que podría caer una nación; el comercio facilita y acerca al progreso".

Esta idea de relevancia del comercio en el trato internacional era lo que primordialmente esgrimían los pretendientes a las cátedras que habrían de ser cubiertas. Algunos mostraban su sentimiento de orgullo:

(1) Archivo General de Indias (en adelante A.G.I.), Sección Consulado, legajo 83. La mayor parte de la documentación consultada para este trabajo procede del legajo citado; cuando esto no sea así lo señalaremos en nota. Sobre este tema también está escrito GARCIA FUENTES, Lutgardo: "La Academia Mercantil del Consulado de Cádiz (1799-1837): Aspectos pedagógicos". En Andalucía y América en el s. XIX, 1986, pp. 303-316. Tomo I. 
"de poder formar parte de este noble grupo, de tener el honor de acercarse con tan noble reto, empleando sus tareas en un obsequio del bien público".

Junto a la Escuela se disponía que funcionara la Sociedad de Ciencias y Artes del Comercio, con el objeto de poner a los estudiantes en contacto con casos reales de la vida comercial.

Para octubre de 1804 había fijado el Consulado el inicio de las clases. Pero no ocurrió así (2), debido a la declaración de guerra con Inglaterra, y los efectos que ésta tendría sobre el comercio, y por otro lado a la aparición de un nuevo brote de fiebre amarilla. Por lo tanto no será hasta enero de 1819 (3) cuando comienzan prácticamente las clases.

En el trabajo que abordamos sólo contemplaremos lo que es fundación y primera etapa de la Escuela Mercantil. Ello no impedirá que en determinados puntos hagamos referencia a hechos posteriores.

\section{EL EDIFICIO}

La primera cuestión a debatir sería el lugar donde se ubicaría esta Escuela de Estudios Mercantiles. ¿En la casa en que en ese momento estaba el Consulado o en otro edificio?, pues el Reglamento, que había de regir el funcionamiento de la Escuela, disponía:

"Estas cátedras ínterin no se construya la Casa Consular según se proyecta, podrán situarse en una particular que se arriende" (4).

(2) FERNANDEZ DIAZ, Roberto; TINOCO RUBIALES, Santiago: "Formación Profesional y Desarrollo Económico: Los Consulados de Cádiz y Sevilla (1784-1829)". Actas del II coloquio de H. ${ }^{a}$ de Andalucía. Córdoba, 1980, p. 224. ...el profesor de la Cátedra primera de 1804, don Joaquín Riquelme, exponía las razones que en su opinión impidieron la apettura de la Escuela Mercantil: "El Plan formado al efecto estaba aprobado por el Gobierno, el edificio que había de servir al intento se hallaba concluido, los profesores nombrados y todo indicaba una próxima apertura; cuando declarada la guerra con Inglaterra, se vio paralizado el comercio de esta plaza, e imposibilitado el Tribunal de destinar a este objeto las grandes sumas que necesitaba, tanto por lo que disminuían sus ingresos, cuanto por las muchas obligaciones que se vio precisado a contraer para satisfacer los grandes empréstitos en que estaba empeñado este comercio para subvenir a los gastos de esta guerra marítima".

(3) Ibídem. El Diario de Cádiz (10-XII-1818), Cádiz. Anunciaba para el día 2 de enero la pública apertura de las clases de Matemáticas y comercio, establecidas por el Real Tribunal del Consulado.

(4) Reglamento del Plan de Estudios de la Escuela Mercantil; 3. ${ }^{a}$ clase. Aranjuez 11 de mayo de 1803. 
La solución fue fundarla en una propiedad del Real Tribunal del Consulado. Propiedad que había pertenecido a la Real Hacienda, y el Consulado la recibió como pago o compensación de un empréstito que realizara a aquella; en el n. ${ }^{\circ} 68$ de la calle San Francisco esquina Aduana Vieja, donde anteriormente hubo una posada conocida con el nombre de "Caballo Blanco", que para ese momento estaba muy deteriorada.

Se pensó que éste sería mejor sitio para ubicar la Escuela que la otra esquina del edificio que ocupaba el Consulado, como también se había propuesto, porque ahí quedaría muy estrecha.

\section{OBRAS}

Para controlar la obra de restauración del edificio de la casa escolar se eligió una comisión en la Junta de Gobierno del 11 de julio de 1803, formada por Francisco Bustamante y Guerra, Francisco Pastor y Calle y Francisco del Valle. Esta comisión gestionó hasta el 1 de abril de 1805 en que se nombraron como sucesores a D. Simón de Agreda y D. Lorenzo de Vitoria, terminando su función con el fin de las obras -no de todo el proyecto del Consulado- de la Casa Academia Mercantil, el 22 de septiembre de 1806.

La dirección de la obra estuvo a cargo de D. Pedro Angel de Albisu. Este arquitecto dirigió otras obras importantes que se realizaron en Cádiz durante este período (5).

Para la restauración y adecuación del edificio surgieron varios problemas. Primeramente nos encontramos con una proposición de los tres primeros comisionados al prior y cónsules del Real Tribunal del Consulado de Cádiz, en donde se expone que:

"al ser aquella casa donde se quiere establecer la Escuela Mercantil tan pequeña que no se alcanza hacer las distribuciones, $y$, al faltar sitio para la escalera, esta se situará en una casa inmediata; pero con esto solo se podría arreglar el repartimiento de aulas y

(5) FALCON MARQUEZ, Teodoro: "Biografía de Pedro Angel Albisu, Arquitecto Mayor de Cádiz". En Homenaje al profesor Carriazo, Tomo III. Sevilla, 1972. También de Pedro Angel Albisu son los planos de las Casas Consistoriales de nuestra ciudad, aunque más tarde sería sustituido por D. Torquato Benjumeda, que con algunas variaciones la continuó. Otros trabajos suyos fueron: el seguimiento de las obras del Ayuntamiento de San Fernando; obras de urbanismo en Jerez en 1784; un pantano en Lorca en 1792 y en 1803 el matadero municipal de nuestra ciudad. 
oficinas de la Escuela, faltando lugar donde ubicar la sala y secretaría para las Juntas de la Sociedad" (6).

Para subsanar estos problemas se consiguió en primer lugar que don Josef Joaquín Montalbo y Sescano, marqués de Casa Tabares, dueño de la casa contigua, la n. .67 de la calle S. Francisco, cediera un patinillo para agrandar la edificación; siendo este espacio el ocupado por la escalera a la que no se le encontraba ubicación en el solar primitivo.

Respecto al segundo problema como los comisionados carecían de facultades para disponer la compra de edificios, proponen al prior y cónsules una solución: el arriendo o adquisición a censo de la casa pequeña contigua a la Academia. Según parece estos cónsules consideraron que con la compra de esa casa quedarían completos los dos establecimientos: de Enseñanza y la Sociedad Mercantil.

Por supuesto los comisionados eran conscientes de los altos costes que esto suponía, pero se atenían a la idea de lo mandado por S.M.:

"que la Academia se fije en la Casa Consular y Lonja que se labre".

Todo esto se unía a la necesidad de construir un edificio nuevo para el Consulado, imprescindible para la comodidad del comercio. Por lo tanto, la ocasión era la indicada para comprar la casa contigua en donde se establecería la Sala y Secretaría de Juntas de la sociedad. Preveiendo el futuro, se dispuso que el edificio que se había de restaurar dedicado a la Academia, se alineara con el que ocupaba el Tribunal del Consulado. De esta forma sólo quedaba una casa intermedia entre ambas, que sería fácil adquirirla por censo. La intención del Consulado era construir un gran edificio, para el que disponía de suficiente terreno, y ubicar, no sólo la Escuela Mercantil y el Tribunal, sino también la Lonja. La construcción se pensó que ocupara toda la manzana, y que fuera con igual decoración que el trozo de la fachada terminada (7). Este edificio, para el que incluso se hicieron planos y se dibujó alzado

(6) La Sociedad de Ciencias y Artes del Comercio estaba íntimamente unida a la Escuela, como expondremos al hablar del Reglamento.

(7) La población gaditana mayoritariamente dedicada al comercio no logró una ciudad tan espléndida como ocurrió en otras de tradición mercantil. El motivo es que al ocupar una pequeña península hay escasez de terrenos. Por lo tanto es difícil planear construcciones que luzcan ante una plaza o una calle de amplias dimensiones. Ello no impidió que se levantaran en Cádiz edificios de buenas proporciones y elegancia.

El gran proyecto de un solo edificio que albergara todas las dependencias consulares hubiera sido una joya del neoclásico. 
resultaría de carácter noble y sencillo con buenas proporciones en sus cuerpos. Su fachada exterior con ventanas y libre de balcones y ornatos daría una perspectiva elegante y bella (8).

El conjunto ubicado en un sitio tan cómodo y céntrico de la ciudad cubriría sus fines positivamente dada la necesidad y utilidad de sus gestiones (9). Es por ello, por lo que los comisionados le piden al prior y cónsules que hagan esta súplica al Rey.

Fueron interesantes las soluciones arquitectónicas que D. Pedro Angel de Albisu dio contra las agresiones climatológicas propias de la ciudad. Dispuso sistemas de protección tanto en escaleras como en azoteas.

Respecto a la ornamentación cabría destacar los 20 balconcitos que daban al patio y escalera. En su cobertura se utilizó piedra de Estepa que había sobrado de la construcción de la Catedral Nueva; en total 249 pies cúbicos. También en la obra se emplearon 278 pies cúbicos de jaspe que decoraron las labores de los pasamanos, mesetas y alicatados de la escalera principal.

Otro material utilizado para 4 columnas de la casapuerta, para las pilastras con sus basas y losas, y para los pasos de la escalera fue el mármol; también se utilizó canto rodado y grandes piedras procedentes de las canterías de El Puerto de Santa María y la Isla de León, especialmente para la construcción de las esquinas del edificio.

\section{FINANCIACION DE LA FUNDACION}

Fue necesaria la contratación para la obra de: albañiles, carpinteros, aserradores, canteros, raspadores y acarretos, como lo prueban las numerosas cuentas que aparecen en la documentación. Para dicha obra también se empleó: madera, cal, ladrillo, cristales, clavos, arena, yeso, etc. (10).

(8) SISTO VACCARO, Tomás de: "Colección de vistas, iluminadas, de los principales edificios de Cádiz, con una breve noticia de su fundación, destino y mérito artístico de sus fábricas". Publicado por la Real Academia de Bellas Artes de Cádiz. Edición facsímil. Cádiz, 1989. Este libro contiene una vista del alzado de la Escuela de Comercio, proyecto que nunca se realizó.

(9) Definitivamente, el edificio para la Escuela Mercantil comenzó a labrarse en la esquina de la C/. San Francisco con C/ Aduana Vieja. Pero las obras para la edificación que debían albergar todas las dependencias del Consulado nunca se llevaron a cabo, debido a la falta de fondos que se habían previsto como necesarios para su construcción.

(10) Elementos que también son registrados en la documentación: esparto, sierra, cal viva, brocha, agua, cola, plomo, papel cortado, junto a gastos de mandados. 
De resultas, desde que empieza la obra el 2 de enero de 1804, hasta el 30 de marzo de 1805 -fecha en que cesa la primera comisión formada por Francisco Bustamante y Guerra, Francisco Pastor y Calle y Francisco del Valle- se gastan 757.016 reales y 20 maravedises de vellón. Desde la última fecha hasta el 22 de septiembre de 1806 en que finaliza la obra, período del que lleva la contabilidad la segunda comisión -formada por Simón de Agreda y Lorenzo de Vitoria- se gas$\tan 360.834$ reales y 20 maravedises de vellón. Para estos gastos han tenido unos ingresos: en la primera etapa 758.111 reales y 20 maravedises, y en la segunda: 380.421 reales y 20 maravedises.

Como podemos comprobar siempre hubo saldo favorable, 1.095 reales de vellón como resulta de la primera gestión y 19.587 reales de vellón de la segunda.

El Consulado tuvo gran interés en que triunfara el proyecto de la Escuela Mercantil; por ello facilitó todas las medidas que supusieran entradas económicas:

1. Dinero procedente de la venta de materiales sobrantes de la construcción de la Catedral Nueva de Cádiz. El Consulado pudo tomar esta decisión debido a que fue el primer contribuyente en la construcción de dicho monumento. Por ello ahora podía disponer de la venta de estos materiales.

2. Alquileres a particulares de dependencias o accesorias de su propiedad.

Para 1804, año en que comienzan las obras para la restauración del edificio de la Escuela Mercantil, son 6 las accesorias alquiladas a particulares, mientras que para 1806 eran ya 11 las accesorias alquiladas y un almacén.

De estas 11 acesorias, 5 se encontraban ubicadas en la C/ Aduana Vieja, posiblemente en la casa n. ${ }^{\circ} 25$, también propiedad del Consulado; las otras 6 se situaban en la C/ San Francisco (11).

(11) Cuadro de localización de las puertas de las distintas accesorias en 1809.

\begin{tabular}{|c|c|c|}
\hline accesoria .. & 2 puertas & C/ San Francisco \\
\hline 1 accesoria & 2 puertas ... & C/ San Francisco \\
\hline 1 accesoria .......... & 1 puerta ..... & C/ San Francisco \\
\hline 1 accesoria ... & 1 puerta ...... & C/ San Francisco \\
\hline accesoria $\ldots$ & 2 puertas ... & C/ San Francisco \\
\hline accesoria. & 4 puertas & $\begin{array}{l}1 \mathrm{C} / \text { San Francisco } \\
3 \mathrm{C} / \text { Aduana Vieja }\end{array}$ \\
\hline accesoria & 2 puertas ............................... & C/ Aduana Vieja \\
\hline accesoria & 1 puerta & C/ Aduana Vieja \\
\hline cesoria.. & 1 puerta & C/ Aduana Vieja \\
\hline cesoria & 2 puertas & C/ Aduana Vieja \\
\hline cesoria & & C/ Aduana Vieja \\
\hline
\end{tabular}


Según la documentación anual que se llevaba, sabemos que fueron numerosos los rendimientos que produjeron estos alquileres; así por ejemplo los precios de los arrendamientos en torno a 1804-1805 de las accesorias situadas en la C/ San Francisco rondaron los 20 a 30 pesos de 19 reales de vellón, mientras que los de la C/ Aduana Vieja fueron alquileres más bajos.

Sería curioso de señalar las condiciones que se imponen en los contratos de arrendamiento a los particulares que alquilaban dichos locales:

"1. Se deberá tener siempre pagado el dinero en efectivo de un mes por adelantado al Real Tribunal del Consulado. alguno.

2. No se pondrá en la calle ni fuera moches ni colgajo

3. No harán ruidos que puedan incomodar a los estudios de dicha casa, ni más fuego que el de uso doméstico.

4. Siempre que se faltara a alguna de las cláusulas, tendrá dicho Real Tribunal acción para despedirme y yo obligación de dejar desocupada la casa a los 15 días de la primera intimación que se haga".

3. Otros tipos de entrada de diversas procedencias.

A pesar de esta situación aparentemente excelente desde el punto de vista económico, hay que hacer referencia a los innumerables gastos que se producen con las reformas del edificio para albergar la Escuela Mercantil. Así pues, habría que mencionar la existencia de unos presupuestos iniciales, que no se ajustan a la realidad a medida que los proyectos se van llevando a cabo. Encontramos numerosas peticiones solicitando aumentos de presupuestos (12).

\section{MANTENIMIENTO ECONOMICO DE LA ESCUELA}

Para el posterior mantenimiento económico de la Escuela, los métodos que se utilizaron podríamos resumirlos, a los siguientes: las entradas de dinero siguieron basándose principalmente al igual que en su fundación, en el producto del arrendamiento que percibían de las 11 accesorias y un almacén, alquilados a particulares.

(12) Por ejemplo, el 27 de octubre de 1804, encontramos una petición de los tres primeros comisionados al prior y cónsules para que se le aumente en 8.000 pesos los presupuestos para hacer la escalera. 
Otro ingreso para el mantenimiento de la Escuela fue el producto de la venta de agua del aljibe de la casa de la Escuela Mercantil, del que encontramos muchos justificantes (13). Una excepción es la de 1818 en que por orden del Sr. prior se prohíbe la venta de agua del aljibe:

"el año aparentaba ser de cortas aguas por lo que podría ser necesitadas para ellos".

Después de examinar numerosos documentos viendo los gastos e ingresos que se producen en el funcionamiento de la Escuela Mercantil; creemos que el Consulado consiguió que la Escuela fuera totalmente autosuficiente, y sólo en última instancia el Consulado tuviera que hacer algunos pagos. Por lo tanto con lo dicho, nos afirmamos en la idea de que se pretendió crear una Escuela que no fuese gravosa al Consulado, sino que pudiese autofinanciarse por sí misma.

\section{LOS ESTUDIOS}

Para acceder a comprender el funcionamiento de la Escuela Mercantil, desde el punto de vista académico, consideramos prioritario el estudio de su Reglamento.

Hemos de destacar al doctor D. Francisco Javier Díaz Cate como autor del Plan de Educación de la Escuela Mercantil, y de las normativas que regirán la Sociedad de Ciencias y Arte del Comercio. Cuyos planes, expuestos al Rey en 1799, fueron aprobados en mayo de 1803 en los mismos términos en que les habían sido propuestos.

Como bien se reflejaba en el Reglamento, el objeto de la enseñanza era:

"formar comerciantes instruidos, que sepan calcular con rectitud, negociar con conocimiento y extender sus ideas sobre incontestables máximas, que aseguren el acierto. Una práctica no ilustrada con oportunas teorías es una ceguedad, en que por acaso se prospera, pero se yerra por necesidad y nunca se adelanta".

\section{Plan de Educación}

El "Plan de Educación para aprender comercio por principio que perfeccione la profesión y faciliten su progreso" estaban compuesto de:

(13) Tenemos constancia que en este momento se sacaba de esta venta un total de 100 pesos de a 15 reales de vellón. 
Introducción: En ella se señalaba la importancia de un buen conocimiento mercantil para estimular y promocionar el desarrollo del comercio. Para ello habrá que acudir a las siguientes cátedras.

Primera Clase: Matemáticas. Para matricularse en ella había que superar las pruebas de gramática castellana, buena letra y las cuatro reglas de cálculo.

Se enseñaban matemáticas más elevadas, el valor y relación de monedas propias y extranjeras, y contabilidad. Se harían ejercicios prácticos de estas operaciones.

Segunda Clase: Relaciones comerciales entre España y el extranjero. Para acceder a ella se requería haber superado la primera.

Se señalaba el conocimiento de la esfera terrestre y los puertos más importantes para el comercio mundial, la calidad de las distintas fabricaciones y manufacturas que en ellos se producían, su transporte y calidad. Por último, se instruiría en los impuestos que gravaban la importación y exportación de géneros, así como a extender facturas y notas.

En esta segunda clase también se impartirían prácticas en las que se mostraría las características de géneros y frutos, de forma que los conocimientos adquiridos no fueran puramente teóricos.

Tercera Clase: Legislación Mercantil en España y el extranjero y la importancia y necesidad del comercio en un estado. Con esta Cátedra se intentaba completar las enseñanzas de las dos anteriores que se exigía que estuviesen superadas. También habría clases prácticas.

La duración de los estudios sería de dos años.

Esta enseñanza se complementaría con la de tres maestros, de francés, de inglés y de toscano. Los idiomas van tomando cada vez mayor importancia, pues eran considerados conocimientos necesarios para los futuros comerciantes (14), de hecho en el Reglamento de 1837 se convierten en una nueva cátedra.

Los maestros ocuparían su cargo previo examen. La vigilancia de su cumplimiento correría a cargo de los diputados del comercio que apercibirían cualquier defecto a la Junta de Gobierno.

(14) Esta relevancia de los idiomas es propia de la época, así la vemos también reflejada en el Real Colegio de San Telmo de Málaga. BORREGO PLA, M..$^{\underline{a}}$ del Carmen: "El Real Colegio de San Telmo de Málaga a través de las Ordenanzas de 1789", en Andalucía y América en el s. XVIII. Escuela de Estudios Hispanoamericanos. Consejo Superior de Investigaciones Científicas. Sevilla, 1985, tomo II, pp. 151-172. Y en el Plan de Enseñanza realizado por el Consulado de Sevilla en 1818. FERNANDEZ DIAZ, Roberto; TINOCO RUBIALES, Santiago: "Formación Profesional y Desarrollo Económico: Los Consulados de Cádiz y Sevilla (17841829)". En Actas del II Coloquio de H. ${ }^{a}$ de Andalucía. Córdoba, 1980, p. 228. 


\section{Horarios}

Los horarios de la Escuela serían en invierno de 9 a 12 de la mañana, y de tarde de 3 a 4,30. En la estación media de 8 a 11 de la mañana y de 4 a 5,30 de la tarde.

\section{Alumnos}

Los alumnos para ser admitidos en el primer curso tenían que tener el visto bueno del prior, cónsules y Diputación y superar los exámenes de gramática castellana, escritura y las cuatro reglas. También podría ser admitido algún comerciante como libre oyente.

Los alumnos estaban obligados a asistir diariamente a clase, a no ser que hubiese causa justa que supiera el director; si la ausencia pasaba los 6 días, se daría cuenta a la Comisión para que ella diera solución.

\section{Gastos}

Todos los gastos -sueldos de los maestros, arrendamientos de las casas y preparación de las clases- se costearian de los fondos del Consulado, según la Real Orden del 26 de febrero de 1799.

\section{- La Sociedad o Academia de Ciencias y Arte del Comercio}

En el impreso, a continuación de este Reglamento, aparecía la normativa por la que se regía la "Sociedad o Academia de Ciencias y Arte del Comercio"; Sociedad que se suponía que contribuiría al éxito de la Escuela. Destaquemos el primer punto que rige la Sociedad:

"No tiene costo este establecimiento y es el medio más a propósito para conseguir adelantamientos".

Con esta Sociedad se pretendía que los expertos en el comercio internacional matriculados en el Consulado de la ciudad gaditana, contaran sus experiencias mercantiles de forma que los alumnos las pudieran incorporar a sus conocimientos (15). Sólo en el caso de que algún socio tuviera que superar algún obstáculo y su aportación supu-

(15) FERNANDEZ DIAZ... p. 623... "Realmente este doble proyecto de Escuela y Academia de Comercio intentaba combinar, igual que en Barcelona... una división del trabajo cultural por la que la Escuela enseñaba y la Academia se encargaba de investigar". 
siese un notable beneficio para el comercio, el presidente y vocales de la Sociedad podrían otorgarle una compensación decorosa de los fondos del comercio.

Como hemos dicho más arriba, este primer Reglamento data del 11 de mayo de 1803, y hasta 1837 no aparece un nuevo "Reglamento del Gobierno Interno de la Academia Mercantil". A partir de ahora, irán apareciendo sucesivas reglamentaciones de distintos años, en donde se le añaden reformas o ampliaciones a algunos artículos, $\mathrm{u}$ otros nuevos.

Es interesante fijarse en los distintos nombres que van tomando estos estudios a lo largo del siglo XIX; así por ejemplo en:

-1803: Escuela Mercantil del Consulado de Cádiz.

-1819: Escuela de Matemáticas y Comercio del Real Tribunal del Consulado de Cádiz.

-1854: Escuela Industrial de Comercio y Náutica.

-1861: Escuela Industrial y de Comercio y Profesional de Náutica.

En esta evolución que sufren los nombres, es significativo ver como los estudios de náutica van tomando personalidad propia, y se van independizando de lo que en un principio era la Escuela Mercantil, donde se empezaron impartiendo unas simples nociones de pilotaje.

\section{EL PROFESORADO}

Para la ocupación de las nuevas cátedras de la Escuela Mercantil, son innumerables las solicitudes que llegan de muchos lugares de España (16); esto nos puede demostrar la importancia que tuvo la creación de dicha Escuela, para la ciudad, en ese período.

(16) Ofrecemos aquí una lista de algunos aspirantes a cátedras en 1803:

\section{Nombre}

D. Gregorio Cabañas

D. Pedro Borel

D. Francisco Guardia

D. Esteban Narice

D. Joaquín de Riquelme

D. Andrés Ortiz de Zárate

D. Luis Astigarraga

D. Gaspar Bastaeli

D. José Antonio Chamorro

D. Agustín Fernández de Espinosa

D. Claudio M. ${ }^{\mathrm{a}}$ Laudriec

D. Francisco Moreno y Herrera

D. Pedro Pardo Santayana

D. Francisco de Biroteau

\section{Cátedra o Clase a la que aspira}

1. a Cátedra

1. ${ }^{\text {a Cátedra }}$

1. Cátedra

1. ${ }^{\text {a Cátedra }}$

1. a Cátedra

1. átedra

2. ${ }^{\text {a }}$ Cátedta

2. ${ }^{a}$ Cátedra

2. ${ }^{\text {a }}$ Cátedra

2. ${ }^{\text {a }}$ Cátedra

2. Cátedra o Clase de idioma francés

2. ${ }^{\text {a }}$ Cátedra

2. a Cátedra

Clase de idioma francés 
En cuanto al tipo de oposición al que tenían que concurrir los candidatos al profesorado, se decidió que para evitar gastos y problemas entre los numerosos solicitantes, se convertiría la oposición o examen en una operación muy sencilla. Consistía en remitir cada uno el Tratado o proyecto relativo a las clases que aspiraba dar con una exposición de sus méritos, e indicaciones personales que pudiesen dar informes. Así pues, a partir de ahora comenzarán a llegar todas estas solicitudes -27 en total-, junto a innumerables certificaciones de profesores y personajes destacados en las materias competentes. En éstas, se alababa o certificaba los estudios, sabidurías, trabajos anteriores, y los méritos que dichos aspirantes a cátedras habían obtenido, con la sola intención de hacer mayor presión en el futuro nombramiento de estos candidatos a las cátedras.

Siempre se tuvo muy en cuenta el saber elegir a los profesores adecuados; y así lo recuerda un escrito en que aparecen estas frases:

"No todos los profesores son buenos, hay que saber elegir a los correctos, teniendo en cuenta que luego tengan las mismas ideas e inquietudes pues luego puede que surjan diferencias y discusiones entre las cátedras y no haya la unidad de enseñanza deseada que sea capaz de crear individuos únicos y fuertes capaz de llevar a cabo este oficio de comercio con grandeza para nuestro país".

Aparecen gtros documentos donde se repite la importancia de saber elegir a los profesores, no solo en el aspecto de tener una semejanza de ideas y fines, sino también de disponer de una mente abierta y dispuesta a la práctica de esa enseñanza (17), ya que para la época:

"de la piráctica acertada proviene la subsistencia y fortuna de millares de individuos construyéndose la más fuerte columna en cualquier República o Monarquía".

D. Agustín Chateau Neuf

D. Pedro Fuertes

Clase de idioma francés

D. Félix de Marcout Clase de idioma francés

D. Andrián Morieau Clase de idioma francés

D. Juan Campel Clase de idioma francés

D. Luis Servientis Clase de idioma francés

D. Carlos Savi Vilalta Clase de idioma francés

D. Pedro Jacome Clase de idioma inglés Clase de idioma toscano

(17) Desde que comienzan los planes para el establecimiento de la Escuela Mercantil esta idea es ya concebida. 
Se establece que los catedráticos tendrían un sustituto que le auxiliaría y ayudaría en la materia, tanto en el caso de tener mucho trabajo como en el caso de enfermedad o cualquier otra necesidad.

Respecto a los textos que estos profesores utilizarían en sus clases, el Reglamento indicaba que a pesar de existir grandes obras de célebres autores sobre las materias que se explicaban, los catedráticos escogieran las partes que ellos considerasen más convenientes de cada obra y formaran un cuerpo doctrinal -un tratado-, más pequeño y comprensible, y ya más tarde se publicarían Ordenanzas con las materias que se leerían en el aula (18).

Sabemos que la enseñanza fue gratuita, y así se expresará más detenidamente en el Reglamento de 1837:

"Opúsculo que publica la Junta de Comercio de esta plaza, con relación a los actos de oposición a las dos nuevas Cátedras que ha establecido en su Academia de Enseñanza Gratuita e inauguración de ellas, en el cual se contienen discursos luminosos y de recomendables doctrinas para el desarrollo del comercio, fuente de felicidad pública. Cádiz 1837".

Se preveía que los sueldos que correspondieran a estos profesores y a sus ayudantes fueran:

Primer catedrático

Ayudante

Segundo catedrático

Ayudante

Tercer catedrático

Ayudante

Maestros de idiomas
1.000 reales mensuales 370 reales mensuales 100 pesos de vellón mensuales 50 pesos de vellón mensuales 1.000 reales mensuales 370 reales mensuales 50 pesos mensuales

Había otros cargos que contribúan al funcionamiento de la Escuela como eran: el administrador cuya función consistía en encargarse del cobro de los alquileres de las accesorias y el almacén y entregarlo a la tesorería del Tribunal; los mozos de casa, que asistían día y noche, encargándose de mantener el aseo de la casa y el tesorero.

(18) En el artículo 6 del Reglamento del Gobierno Interior de la Academia Mercantil de 1837, se establecía la libertad de los profesores para elegir textos de autores y métodos de enseñanza, siempre que dieran cuenta de esta elección a la Junta de Comercio para su aprobación. 


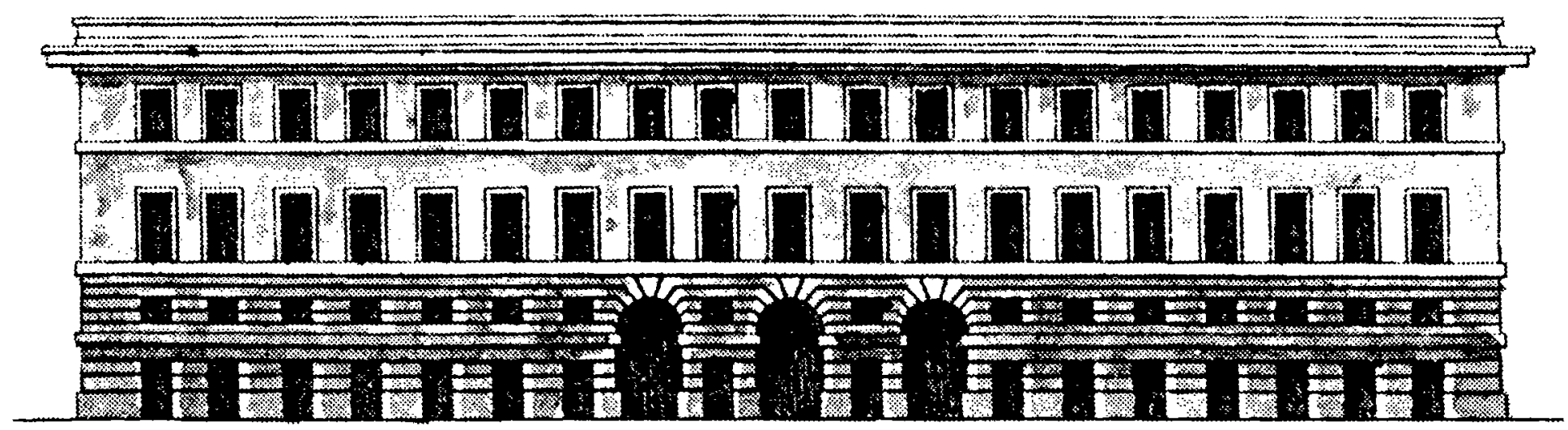

ESCUELA DE COMERCIO 